\section{Sensibilitätsstörungen beachten}

_ In Deutschland werden derzeit pro Jahr 40.000 Amputationen infolge eines diabetischen Fußsyndroms durchgeführt, so der Internist Dr. Arthur Grünerbel vom Diabeteszentrum München-Süd. Dies sei umso bedauerlicher, als diese Katastrophe einen längeren neuropathischen Vorlauf habe und durch rechtzeitige Gegenmaßnahmen abgewendet werden könne.

Gewissermaßen „leider“, so Grünerbel, fällt eine diabetische Neuropathie nur bei einem Viertel aller Betroffenen mit Schmerzen auf, die stark genug sind, um die Patienten rasch zum Arzt zu führen. Weniger spürbare Warnsignale bis hin zu manifesten Druckgeschwüren werden oft lange übersehen, unter schätzt oder ignoriert. Umso wichtiger ist es, dass Ärzte bei Diabetikern gezielt nach suspekten Symptomen fragen und die Füße regelmäßig gewissenhaft auf Sensibilitätsstörungen und konsekutive Läsionen untersuchen. Diesbezüg-

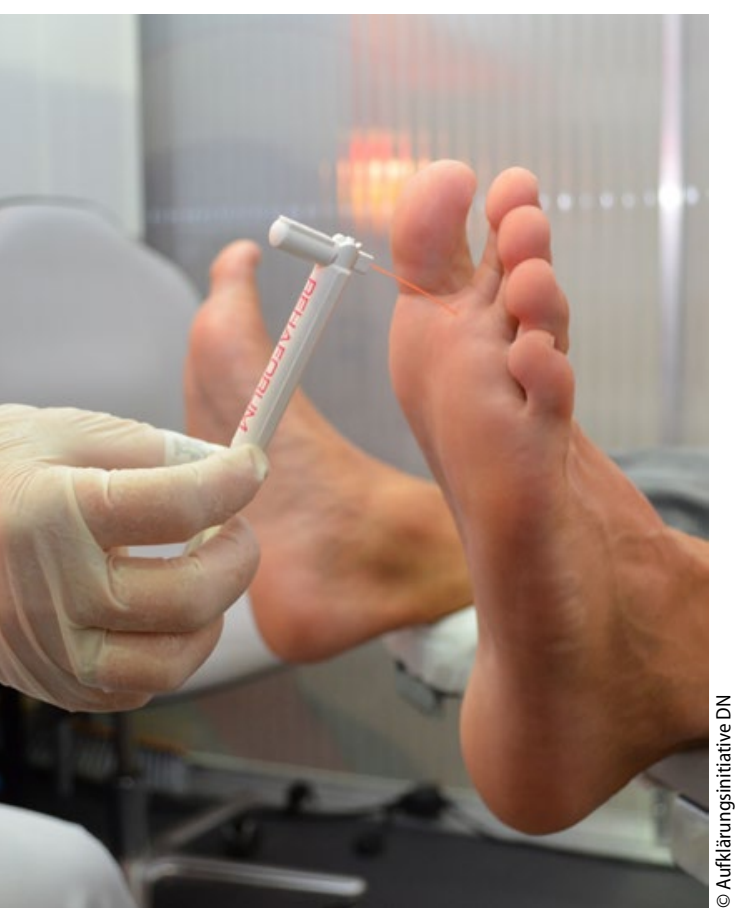

Regelmäßige Fußuntersuchungen sind bei Diabetikern ein absolutes Muss. lich scheint Deutschland allerdings noch ein Entwicklungsland $\mathrm{zu}$ sein. Grünerbel zitierte Ergebnisse der nationalen Kora-F4-Studie, wonach bei jedem dritten der dort erfassten über 60-jährigen Diabetiker noch nie die Füße inspiziert worden waren.

\section{Interdisziplinäre Fußrettung}

Eine diabetische Neuropathie ist eine sehr häufige Diabeteskomplikation. Etwa jeder dritte Zuckerkranke ist betroffen. Speziell bei Typ 2-Diabetikern etabliert sich das Nervenleiden gar nicht so selten, noch bevor die Zuckerstoffwechselstörung diagnostiziert ist - oder, so auch Grünerbels Erfahrung, sogar schon in prädiabetischen Stadien. Die Prävalenz diabetischer Fußulzera in der diabetischen deutschen Bevölkerung werde aktuell auf zwischen $2 \%$ und $10 \%$ beziffert.

Die wichtigste Maßnahme gegen eine diabetische Neuropathie und ihre gravierenden Folgen für die Füße ist eine optimale Blutzuckereinstellung durch Lebensstiländerungen und geeignete Medikamente. Stoffwechselexperten, Neurologen, Angiologen, Orthopäden, Podologen und orthopädische Schuhmacher müssen für eine bestmögliche Versorgung der Patienten zusammenarbeiten.

Ergänzend rät Grünerbel zur Einnahme von Benfotiamin (enthalten z.B. in milgamma ${ }^{\oplus}$ protekt). Das Provitamin gleicht den bei Diabetikern häufigen Vitamin- $B_{1}$-Mangel aus und wirkt der Bildung nervenschädigender Advanced Glycation Endproducts (AGE) entgegen. In einer sechswöchigen, doppelblinden, kontrollierten Studie mit 165 Patienten kam es unter täglich 300 oder 600 mg Benfotiamin gegenüber Placebo zu einer signifikanten Reduktion neuropathischer Symptome insbesondere Schmerzen und Taubheit in den Füßen.

\section{Werner Stingl}

- Media Dinner "Aktiv gegen den Zuckerfuß"; München Oktober 2016 (Veranstalter: Wörwag Pharma)

\section{Patientenleitlinie empfiehlt Johanniskraut}

Nach der Aktualisierung der S3-Leitlinie und Nationalen VersorgungsLeitlinie Unipolare Depression ist nun auch die dazugehörige Patientenleitlinie erschienen. Als wichtigste Behandlungsmöglichkeiten benennt die Leitlinie psychotherapeutische Verfahren und die medikamentöse Therapie mit Antidepressiva. Als einziges pflanzliches Medikament empfiehlt sie Johanniskrautextrakt in ausreichender Dosierung, und zwar für die Indikation unipolare Depression leichter und mittelschwerer Ausprägung.

Red.

- Nach Informationen von Bayer

\section{Postprandialen Blutzucker mit Insulin aspart kontrollieren}

Insulin aspart kann das Risiko für kardiovaskuläre Ereignisse deutlicher reduzieren als kurzwirksame Humaninsuline, wie eine retrospektive Beobachtungsstudie zeigte. So war das Risiko, einen Schlaganfall oder eine transitorische ischämische Attacke zu erleiden, im Vergleich um $42 \%$ geringer. Für einen Myokardinfarkt war es $31 \%$ und für eine periphere arterielle Verschlusskrankheit (PAVK) 20\% niedriger.

Dr. Silke Wedekind

- Springer Medizin Expertenworkshop „Postprandialer Blut zucker - wird seine Bedeutung unterschätzt?"; München, September 2016 (Veranstalter: Novo Nordisk)

\section{Sieben-Tage-Schmerzpflaster mit deutlichen Vorteilen}

Das Sieben-Tage-Pflaster mit dem Wirkstoff Buprenorphin (Norspan ${ }^{\odot}$ ) ist zur lokalen Anwendung bei opioidsensiblen chronischen Schmerzen indiziert und seit April 2016 in den Dosierungen $5,10,20,30$ und $40 \mu \mathrm{g} / \mathrm{h}$ erhältlich. Diese Wirkstärken erlauben einen niedrigdosierten Einstieg und eine bedarfsgerechte Dosissteigerung ohne Präparatewechsel. Das geringe Interaktionspotenzial macht Buprenorphin zum Analgetikum der Wahl bei geriatrischen Patienten. Eine auf www.schmerz-infocenter.de erhältliche Broschüre fasst diese und weitere Vorteile des Schmerzpflasters zusammen. Red. 